\title{
REPEUPLEMENT DES LACS DE MONTAGNE DES HAUTES ET BASSES PYRENÉES ${ }^{(1)}$
}

\author{
par Pierre CHIMITS \\ Ingénieur des Eaux et Forêts à Pau \\ Biologiste des Pêches à l'Organisation des Nations Unies (F. A. O.)
}

Les lacs pyrénéens ont été étudiés systématiquement très récemment et c'est Gaurier qui, en 1930, en fit le premier inventaire géographique et topographique en dénombrant 520 lacs sur le seul versant français des Pyrénées. On peut en supposer un nombre analogue sur le versant espagnol. La plupart de ces lacs sont à haute altitude et presque tous situés entre 1800 et 2500 mètres, c'est-à-dire sous la glace pendant la moitié de l'année au moins. D'autre part, peu de ces lacs renferment du poisson, uniquement la Truite commune avec, parfois, quelques Vairons et il a toujours paru difficilement explicable qu'un lac coupé de toute communication vers l'aval par des chutes infranchissables puisse contenir des Truites, alors qu'un autre situé dans des conditions semblables en contienne.

Les premiers repeuplements artificiels dont j'ai retrouvé trace datent de 1906 : repeuplement du lac de Barroude par Descombes, qui n'a pas réussi. En 1908, Arne repeuple le lac d'Árius actuellement vide de poisson. Le premier effort de repeuplement systématique date de 1936 où M. Larrieu a introduit avec succès Truite arc-en-ciel, Omble chevalier et Saumon de fontaine, notamment au lac d'Oncet où ces poissons se sont fort bien reproduits et maintenus. En 1938, étant l'adjoint de M. Larriev, j'ai aleviné le lac d'Isabe en Omble chevalier qui s'y est également reproduit et maintenu. L'inventaire piscicole de ces lacs pyrénéens des Hautes et Basses-Pyrénées a été exécuté en 1952, grâce à l'aide du personnel forestier et des pêcheurs locaux, et constitue actuellement un document d'autant plus intéressant que, d'une part, l'E.D.F. pour ses travaux, crée des inter-communications entre plusieurs de ces

(1) Conférence donnée le 3 mars 1955 devant la Société Centrale d'Aquiculture et de Pêche. 
lacs qui aboutissent à un mélange de faune et que, d'autre part, depuis 1950, nous avons entrepris le repeuplement systématique de ces lacs.

Cet inventaire porte uniquement sur les lacs des Hautes et BassesPyrénées, ceux des trois autres départements pyrénéens étant actuellement en cours. Il résulte de cet inventaire qu'en 1951, sur 264 lacs de montagne des Basses et Hautes-Pyrénées, 182 étaient sans poissons, soit plus des $2 / 3$. Sur 82 lacs piscicoles en 1951, 34, soit presque la moitié, étaient vierges et ont été peuplés artificiellement par les Eaux et Forêts, dont une vingtaine en 1950 et 1951. Sur les 48 lacs qui semblent peuplés sans origine connue et qu'on peut supposer naturelle, peut-être y en $a-t-i l$ quelques-uns qui auraient été alevinés en Truite commune dans des circonstances inconnues. Certains de ces lacs ont reçu des alevinages complémentaires en Truite arc-en-ciel, Omble chevalier et Saumon de fontaine.

En ce début de 1955, la situation s'est nettement améliorée et le point est le suivant pour ces deux départements : 124 lacs encore vierges, 140 lacs peuplés artificiellement (Truite arc-en-ciel, Truite commune, Saumon de fontaine, Omble chevalier, Cristivomer namaycush et Tanche), dont 92 ces quatre dernières années, ceci uniquement par mon service avec quelques bénévoles de la Fédération de Pêche locale, tous les alevins provenant de la pisciculture domaniale de Cauterets. Sur ces 92 lacs, il est trop tôt pour une cinquantaine environ d'entre eux de parler d'acclimatation réussie, puisqu'il n'y a pas eu encore constat de reproduction naturelle.

TECHNIQUE DU PEUPLEMENT.

Ainsi donc, en cinq ans, 104 lacs pyrénéens vierges ont été alevinés grâce à la pisciculture domaniale de Cauterets remise en service en 1950 et qui déjà compte des reproducteurs et futurs reproducteurs des cinq principales espèces utilisées et énumérées ci-dessus.

C'est donc à la cadence d'une vingtaine de lacs par an que nous avons effectué ces repeuplements. A ce rythme, et compte tenu de quelques échecs qui se produiront et exigeront de nouveaux déversements, il faut compter une dizaine d'années pour donner une population à tous les lacs piscicoles des Hautes et Basses-Pyrénées. Naturellement, un certain nombre de ces lacs, assez faible il est vrai, donneront des échecs, soit en raison de leur trop forte altitude (lac Tourrat) ou de leur trop faible profondeur entraînant, par hiver exceptionnel, un gel total, soit encore en raison de leur exposition aux avalanches, ou pour toute autre cause non définie et probablement chimique (lac d'Antarrouy). Mais il est certain que ce peuplement augmentera fortement les parcours des pêcheurs sportifs et montagnards avec la perspective de beaux paniers. C'est ainsi que, le 14 Juillet 1953, seul avec le guide pyrénéen bien connu, François BOYRIE, de Cauterets, nous avons réalisé, uniquement au lancer léger, un tableau de $22 \mathrm{kgs}$ de salmonides, soit au total 104 pièces (Truite commune, et surtout Truite arc-en-ciel et Saumon de fontaine) dans les 
lacs d'Estibehaute, dans la haute vallée de Cauterets, lacs vierges et alevinés trois ans auparavant par nos soins, et, faut-il le préciser, sans le faire connaître.

Peut-on accroître ce rythme de repeuplement? C'est douteux. En effet, la période pendant laquelle il est possible d'aleviner les lacs est très brève et s'étale de leur dégel (mi-Juin) aux fortes chaleurs (10 au

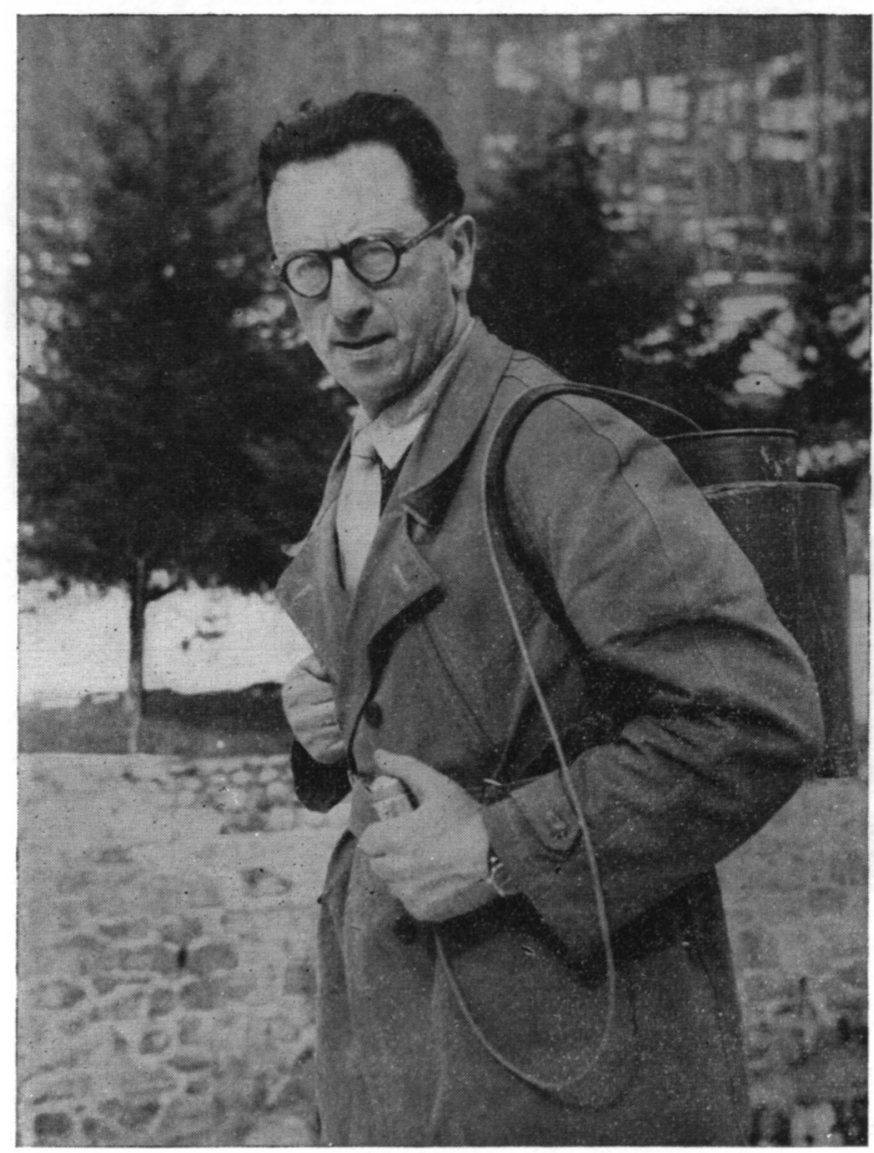

FIG. 1. - Transport d'alevins en montagne.

Bidon da dos et aérateur à main

15 Juillet). Or, nous ne disposons, pour les faire, que de quelques gardes forestiers et de quelques bénévoles effectifs, car si les conseilleurs sont nombreux, rares sont ceux qui acceptent de partir vers les lacs d'altitude, en jeep d'abord, à pied ensuite, pour une saine et tonique marche de quelques heures avec un bidon de 15 à 20 litres sur le dos (fig. 1). En tout cas, la cadence de repeuplement est déjà appréciable et les résultats obtenus intéressants.

Les bidons de déversement en tôle, d'une contenance de 15 litres, 
sont chargés de 3 à 500 alevins de 4 à 5 centimètres de long, quantité qu'il ne faut pas dépasser pour une longue marche. Le bidon est porté à dos, tenu par des bretelles, tout comme un sac de montagne. Si l'on dispose d'un mulet, il peut porter la charge de quatre bidons, deux de chaque côté du bât.

En outre, un petit détail technique permet d'avoir une bonne aération de l'eau dans le bidon : deux petits diffuseurs ronds du modèle vendu chez tous les marchands d'aquariums, sont fixés au fond du bidon et

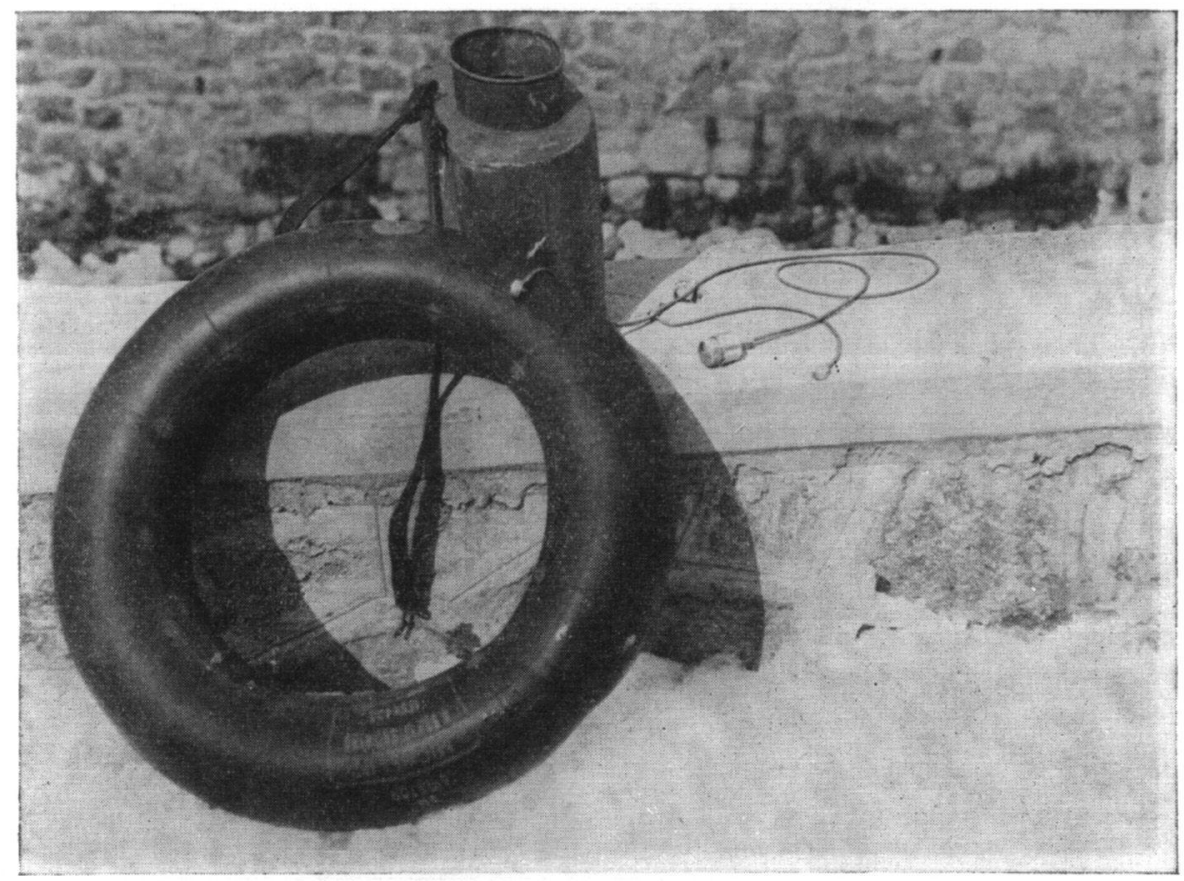

Fig. 2. - Bidon, Aérateur et Diffuseurs.

Lorsque le transport des alevins est effectué à dos de mulet, l'aérateur à main est remplacé par une chambre à air d'auto pourvue d'une valve de gonflage normale et d'un robinet de décharge.

communiquent par un mince tuyau en caoutchouc avec un appareil fournissant l'air. Cet appareil est, soit une chambre à air d'auto placée autour du cou du mulet et que le garde gonfle toutes les demi-heures avec une pompe à bicyclette, soit un petit vaporisateur que le porteur actionne à la main au cours de la marche (fig. 2).

Il faut étudier le parcours à l'avance, de façon à trouver des points d'eau toutes les heures et calculer l'heure de départ de la pisciculture de façon à parvenir au lieu de déversement très vite après le lever du soleil. 
LALTITUDE ET LE REPEUPLEMENT.

Regan indique qu'en Europe la Truite commune n'existe pas audelà de 1.900 mètres. Descombes mentionne le lac pyrénéen de Caillaouas $(2.165 \mathrm{~m}$.) dans la Vallée de la Neste comme le lieu le plus haut habité par la Truite dans les Pyrénées. Dès 1906, Descombes alevine en Truite arc-en-ciel le lac de Barroude (2.359 m. sur la Neste de la Géla) et croit battre le record d'altitude d'acclimatation de la Truite; mais cet essai a été un échec, le lac de Barroude étant actuellement redevenu désert. Roule indique comme limite supérieure de la Truite dans les Alpes le lac d'Allos (2.239 m.) et dans les Pyrénées le lac Lanoux (2.134 m.). Plus tard, il indique comme limite le lac de Couart (2.230 m.). Germain mentionne, sans préciser, l'altitude limite de 2.270 mètres. Enfin, de l'inventaire que nous avons dressé en 1951 , il résulte que la Truite commune existe à l'état naturel aux lacs d'Aygue-Cluse et de Madamette au-dessus de Barèges, à plus de 2.300 mètres et au lac de Baboukou à 2.375 mètres. Toutefois, nous reconnaissons bien volontiers que certains lacs situés vers 2.400 mètres exposés au Nord tels que le lac Noir au-dessus du lac de Cestrède, sont glacés trop longtemps pour que la Truite puisse y vivre; ils ne sont libres de glace sur au moins la moitié de leur surface que pendant trois à quatre mois par an. Le 30 Août 1938, nous avons trouvé le lac Tourrat $(2.619 \mathrm{~m}$.) gelé aux trois quarts avec une température en surface de $1^{\circ} 3 / 4$. Il ne saurait donc être piscicole.

D'autres lacs situés à des altitudes moindres, ne sont cependant pas piscicoles. C'est ainsi que nous avons échoué dans nos essais d'alevinage en Omble et Truite arc-en-ciel du joli petit lac d'Antarrouy (2.000 m.) aux eaux si limpides. Ce lac est très pauvre en nourriture naturelle, pratiquement sans plancton et sans larves d'insectes ce qui semble pour notre échec une raison suffisante; reste encore à expliquer la pauvreté de cette sitèse, alors que le lac voisin de Cestrède est bien pourvu de Truites, tous deux étant également séparés des affluents piscicoles du Gave de Pau par des cascades infranchissables au poisson.

La Truite arc-en-ciel a été introduite au lac Estellat à 2.431 mètres d'altitude et s'y comporte fort bien. Il est curieux de constater qu'il y a une sorte de parallélisme entre les limites supérieures de la végétation forestière et les limites supérieures de vie de la Truite ; cette limite semble être celle du pin à crochets qui est d'environ 2.500 mètres dans les Pyrénées et 2.300 mètres dans les Alpes. Il semble que l'Omble chevalier, et surtout le Cristivomer, puissent être acclimatés à des altitudes légèrement plus élevées.

En résumé, le record d'altitude des salmonides pyrénéens a l'état naturel était 2.320 mètres au lac d'Aygue-Cluse et 2.375 mètres au lac de Baboukou. En 1951, ce record a été porté à 2.431 mètres avec l'acclimatation de la Truite arc-en-ciel au lac Estellat. En 1954, le record a été porté à 2.452 mètres par suite de l'introduction en 1951 de la Truite commune, et de la Truite arc-en-ciel au lac des Oulettes d'Estom-Soubiran; ce record sera peut être battu si l'alevinage effectué en 1954 aux lacs d'Arrailhé, situé sur la route de Vignemale, à plus de 2.500 mètres réussit. 


\section{LES DIVERSES ESPECES HABITANT AGTUELLEMENT LES LACS PYRÉNÉENS.}

La Truite commune existe dans plusieurs lacs à l'état naturel. Adulte, la Truite de ces lacs n'a pas le même aspect que celle des torrents : plus grande, avec une tête plus forte et mieux armée, sa robe comporte de larges taches noires avec très peu ou pas de taches rouges. Roule reconnaît aux Truites de certains lacs de l'Ariège des caractères les rapprochant des variétés endémiques marocaines, avec des yeux grands, des écailles plus petites et plus nombreuses allant jusqu'à 160 sur la ligne latérale. La croissance de la Truite commune dans ces lacs est en général très longue et, en tout cas, bien inférieure à celle de la Truite arc-en-ciel et du Saumon de fontaine quand ils y sont introduits.

La Truite arc-en-ciel, en effet, a donné dans ces lacs et les lacs de barrage d'excellents résultats et une bonne croissance. Au lac d'Ilhéou $(1.976 \mathrm{~m}$.), près de Cauterets, la Truite arc-en-ciel introduite en 1936, se reproduit régulièrement. Au lac d'Estibehaute $(2.360 \mathrm{~m}$.), la Truite arc-en-ciel, après trois ans, pesait de 250 à 400 grammes et était extrêmement grasse, le ventre bourré de phryganes et de coléoptères. La Truite commune, déversée en mème temps, ne pesait que 120 à 150 grammes, alors que le Saumon de fontaine de même âge et de même taille était dans les mêmes conditions physiques que la Truite arc-en-ciel. On peut en déduire que la Truite arc-en-ciel est une espèce plus plastique et capable de s'adapter à des eaux aussi bien plus chaudes que plus froides que la Truite commune. Mais elle supporte mal la concurrence vitale de la Truite commune et sa régénération reste incertaine. Dans les lacs de montagne et aussi dans certains lacs de barrage hydroélectriques, à condition d'opérer des alevinages réguliers, on obtiendra donc d'excellents rendements de poissons poussant vite et ayant une chair grasse et savoureuse avec la Truite arc-en-ciel et le Saumon de fontaine.

Le Saumon de fontaine, ainsi que l'Omble chevalier, a été introduit dans les Pyrénées en 1936 par LARRIEU, gràce aux piscicultures d'Oloron et de Lées Athas. Cette introduction a donné d'excellents résultats et une bonne croissance dans les torrents de haute altitude (Gave d'Aspe, Gave d'Ossau, Haut Adour, Gave de Gaube) et dans certains lacs (Gaube et Estibehaute). Mais, tout comme la Truite arc-en-ciel, si sa croissance est très rapide, sa reproduction naturelle est très incertaine, peut être en raison de sa voracité qui le rend d'une capture facile, et aussi du fait que, comme la Truite arc-en-ciel, il supporte mal la concurrence de la commune. Les lacs où cette espèce a été introduite devront être régulièrement alevinés.

$\mathrm{Au}$ contraire, l'Omble chevalier, se reproduit très facilement dans les lacs pyrénéens mais il donne des populations nombreuses et souvent de petite taille. Nous ne connaissons que deux lacs, il est vrai, grands et profonds, dans lesquels l'Omble chevalier donne des individus dépassant le kg. : le lac de Gaube et le lac d'Artouste. Mais il est à noter que, contrairement à celui des lacs alpins, l'Omble des Pyrénées peut frayer 
dans des lacs peu profonds, de quelques mètres seulement (Isabe, Montagnons d'Iseye, Oncet).

Le Vairon existe dans certains lacs, mais sa présence est artificielle et il n'existe à l'état naturel que dans le lac d'Estaing $(1.170 \mathrm{~m}$.). Introduit il y a vingt ans dans les lacs au-dessus de Barèges, il s'y maintient et s'y reproduit et fournit une nourriture pour la Truite.

Enfin, les deux dernières introductions d'espèces datent de 1953. Le Cristivomer namaycush qui est un Omble des grands lacs du Canada et d'Amérique du Nord, a été introduit dès 1929 avec succès dans quelques lacs Suisses de haute altitude. Par l'intermédiaire de la Station d'Hydrobiologie appliquée, nous avons reçu en 1953, du Canada, 1.200 œufs qui ont été incubés dans les eaux très froides de la pisciculture domaniale de Cauterets. Nous avons mis 600 alevins dans quatre lacs vierges de haute altitude et il nous reste dans les bassins de cette pisciculture 40 Cristivomers actuellement âgés de 2 ans. Au printemps 1954, nous en avons reçu un nouvel envoi de 1.500 œufs avec lesquels nous avons aleviné trois autres lacs et il nous reste environ 500 Cristivomers de 1 an qui constitueront le futur cheptel de reproducteurs de cette pisciculture, à notre connaissance la seule en France et en Europe à contenir des Cristivomers. Ce salmonide au corps très allongé et mince, semble être une excellente acquisition pour les lacs très froids, mais il est encore trop tôt pour parler de son acclimatation puisque sa reproduction n'a pu encore avoir lieu. Au Canada, il ne commence à se reproduire qu'à 7 ans.

La dernière introduction est celle de la Tanche. En Juin 1953, nous avons porté une douzaine de reproducteurs de Tanche provenant de l'étang de Moliets (Landes) qui est situé au bord de la mer, à une altitude de quelques mètres à peine, et les avons placés dans la laquette de Collongue au-dessus de Barèges, à 1.800 mètres, qui est une mare profonde de 2 mètres seulement et glacée l'hiver, mais a une température chaude pendant trois ou quatre mois l'été. Dès l'été 1953, ces Tanches frayaient et la laquette contient actuellement de nombreux alevins de Tanche dont la période active est réduite à quatre mois. La Tanche nous semble donc intéressante à introduire dans les lacs de moyenne altitude car ses alevins, comme le Vairon, pourront servir de nourriture à la Truite, avec, sur le Vairon, l'avantage de ne pas prendre la nourriture de la Truite et de ne pas s'attaquer à ses œufs.

Ainsi donc, nous connaissons les espèces qu'il faut mettre dans les divers lacs d'altitude pyrénéens et savons comment transporter les alevins. D'autre part, la pisciculture domaniale de Cauterets contient dans ses bassins une quantité déjà importante de reproducteurs de ces diverses espèces. L'alevinage de tous les lacs pyrénéens d'altitude des Hautes et Basses-Pyrénées n'est donc plus qu'une question de temps et de persévérance. Il pourrait être considérablement accéléré et facilité par l'emploi de l'hélicoptère, récemment utilisé avec succès dans les chantiers hydroélectriques de haute montagne des Pyrènées. 